www.jmscr.igmpublication.org

Impact Factor 5.84

Index Copernicus Value: 83.27

ISSN (e)-2347-176x ISSN (p) 2455-0450

crossref DOI:_https://dx.doi.org/10.18535/jmscr/v5i5.46

\title{
A Study of Different Hematological Parameters in Malaria
}

\author{
Authors \\ Joshi Hetal J. ${ }^{1}$, Sapre Jyoti $\mathbf{P}^{2}$ \\ ${ }^{1,2}$ Assistant Professor, Department of Pathology, Pramukhswami Medical College, Karamsad \\ Corresponding Author \\ Dr Hetal J Joshi
}

Address: Ashish Hospital, Sherpura, Sardar Chowk, Petlad-388420.District: Anand Gujarat India

Phone numbers: 918156072400, Email: hetaljj@charutarhealth.org

\begin{abstract}
Aims: To study an alteration in hematological parameters in malaria.
\end{abstract}

Settings and Design: This is a prospective study conducted at Central Diagnostic Laboratory attached to Shree Krishna Hospital, Karamsad. All the smears positive for malaria cases, diagnosed at Pathology Laboratory were selected and studied for hematological changes.

Methods and Material: According to Proforma, detailed history regarding age, sex, nature and duration of illness is taken. Venous blood in EDTA vaccutainer was collected from patient for diagnosis of malaria and hematological study before starting anti-malarial treatment.

Results: Out of 51 malaria positive patients, 41(80.3\%) patients had P.vivax infection while 10 (19.6\%) were infected with P.falciparum. Anemia, Thrombocytopenia and leucopenia were observed in 25 (49.01\%), 48 $(94.11 \%)$ and $9(17.64 \%)$ patients respectively.

Conclusions: Species variation is seen in altered hematological parameters. Anemia and thrombocytopenia were more severe with p.falciparum infection while mild to moderate leucopenia was observed in p.vivax infection and it has no relation with p.vivax infection.

Keywords: Malaria, P.falciparum, P.vivax.

\section{Introduction}

Malaria is a mosquito-borne infectious disease of humans and other animals caused by parasitic protozoan's belonging to genus Plasmodium. ${ }^{1}$ Malaria continues to be a major health problem in some of the most populated areas of the world. It is one of the important causes of febrile illnesses in our part of the world. One of the most prevalent human infections worldwide, malaria results in 225 million cases each year. Around $40 \%$ of the global population at risk of malaria resides in the South-East Asian Region. It is caused by protozoa parasite of the genus plasmodium which infects and destroys red blood cells. Four species of plasmodia (P.falciparum, P.malariae, P.ovale and P.vivax) cause malaria in humans of which P. falciparum is the main cause of morbidity and mortality ${ }^{2,3}$. Some hematological changes are species specific. Thrombocytopenia is a common and early sign of malarial infection $\&$ it is observed in vivax and falciparum malaria in varying degrees. Most of these findings are more pronounced in P.falciparum as compared to vivax. ${ }^{4,5}$ However, there is no unanimity in the 
findings with some studies showing minimal differences between the two and others showing a more pronounced hematological alteration in falciparum as compared to P.vivax ${ }^{7,8,9}$. There have also been reports of leucopenia and leukocytosis. ${ }^{10}$

\section{Subjects and Methods}

This is a prospective study conducted at Central Diagnostic Laboratory attached to Shree Krishna Hospital, Karamsad. All the smears positive for malaria cases diagnosed at Pathology Laboratory were selected and studied for haematological changes.

According to Proforma, detailed history regarding age, sex, nature and duration of illness is taken. Findings of clinical examination were noted. Venous blood in EDTA vaccutainer was collected from patient for diagnosis of malaria and hematological study before starting anti-malarial treatment.

Once the blood sample received in the laboratory then kept them on blood mixture, then $\mathrm{Hb}$, Platelet Count and Total WBC Count were done in automated KX-21 hematology analayzer. ${ }^{11}$ After that from same sample thin and thick smears were prepared. Both were stained with giemsa stain. Thick smears were examined for rough estimation of parasite by observing average no.of parasites per oil immersion field. Thin smears were examined for confirmation of Platelet Count and for identification of species and grading of parasitemia.

\section{Results}

The study of hematological changes in malaria is carried out at Shree Krishna Hospital, Karamsad from the period of February 2014 to February 2015(one year). All malaria positive cases were included and Hemoglobin, Platelet count and Total WBC count of all were studied. Following observation from this study has made.

Out of 51 malaria positive patients $37(72.45 \%)$ were male and $14(27.45 \%)$ were female. Majority $(23.52 \%)$ of patients were in the age group of 1120 years. Fever with chills, rigors and headache were the commonest presenting complaints.
41(80.3\%) patients had P.vivax infection while 10 (19.6\%) were infected with P.falciparum infection. From the 51 cases of malaria, anemia was observed in 25 patients (49.01\%). Amongst them $68 \%$ were of P.vivax infection and $32 \%$ were of P.falciparum infection.

Thrombocytopenia was seen in 48 (94.11\%) patients. Amongst them $90 \%$ were of P.falciparum and $95.12 \%$ were of P.vivax infection. Severe thrombocytopenia was seen in $40 \%$ of falciparum and $21.95 \%$ of P.vivax patients.

Leucopenia was seen in 9 patients (17.64\%). Amongst them $10 \%$ were of P.falciparum and $19.51 \%$ were of P.vivax malarial infection. We have received only10 repeat samples during whole study for laboratory investigation. In all repeat samples marked variation in platelet count was observed. Once the treatment was started, Platelet count was increased abruptly, but hemoglobin and total WBC Count were not much affected.

Table Comparision of Mean Hemoglobin, Platelet and WBC Between P.Vivax and P.Falciparum

\begin{tabular}{|l|l|l|l|l|}
\hline $\begin{array}{l}\text { Sr. } \\
\text { no }\end{array}$ & Parameter & P.vivax & P.falciparum & P value \\
\hline 1 & Mean HB & $11.38 \pm 3.58$ & $7.9 \pm 6.0$ & $* 0.015$ \\
\hline 2 & Mean Platelet & $100.21 \pm 153.56$ & $112.02 \pm 73.5$ & 0.384 \\
\hline 3 & Mean WBC & $6.4 \pm 11.14$ & $6.38 \pm 6.70$ & 0.13 \\
\hline
\end{tabular}

*indicates $\mathrm{P}$ value $<0.05$ - statistically significant

- There is a statistically significant differrence in Mean Hemoglobin value between P.vivax and P.falciparum. (P value $<0.05$ ).

- There is no statistically significant difference_in Platelet count between P.vivax and P.falciparum in this study. ( $\mathrm{P}$ value $>0.05$ ).

- There is no statistically significant difference in WBC Count between P.vivax and P.falciparum in this study. ( $\mathrm{P}$ value $>0.05$ ).

Figure:1 Labeled thin blood film (A) and thick blood film (B) 


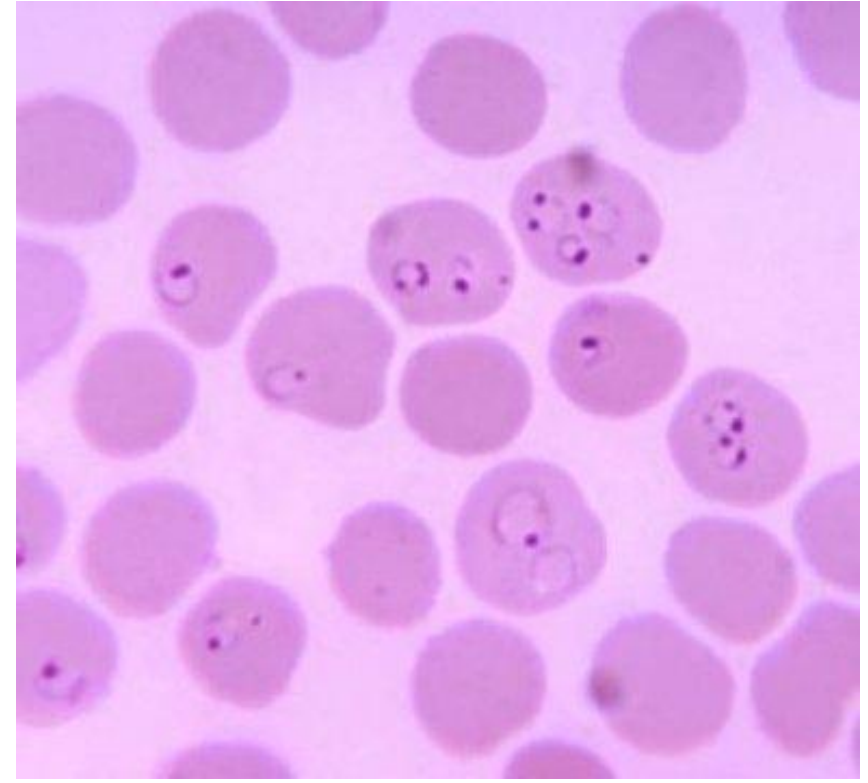

Figure: 2 Mixed Infection (Trophpzoites of P.vivax and P.falciparum) (Thin smear)

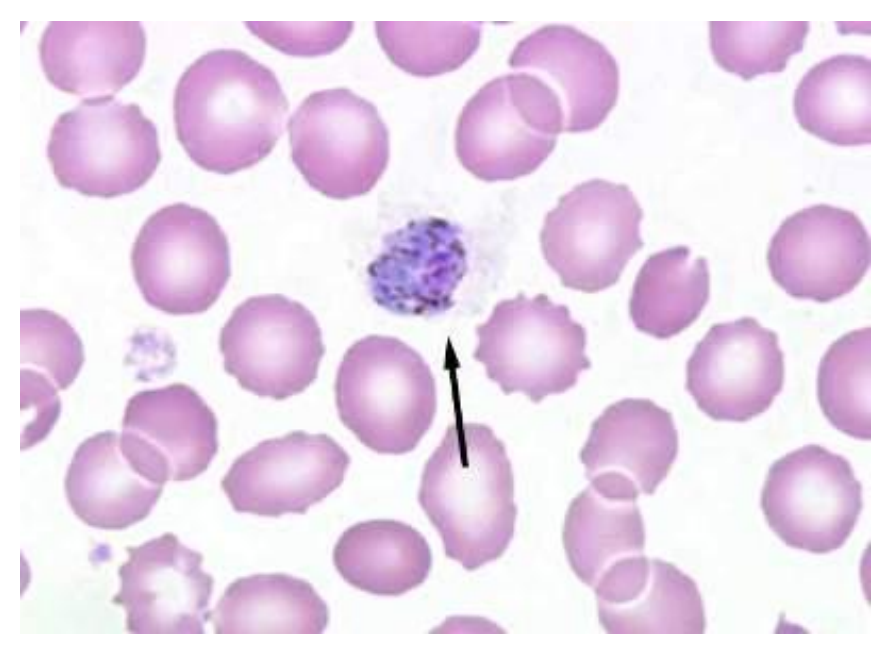

Figure: 3 Schizonts of P.vivax (Thin smear)

\section{Discussion}

Out of studied 51 cases, $41(80.3 \%)$ were of P.vivax and $10(19.6 \%)$ cases were of P.falciparum. This study includes 37 male patients (72.54\%) and 14 females (27.45\%). In the present study male are more commonly affected. These findings are comparable to other study conducted by Ranjini CY et al, who found that male $(80.24 \%)$ was more commonly affected than female $(19.75 \%)^{12}$. Age wise distribution in the present study showed that $72.52 \%$ patients were adults (11-50 years). Majority (23.52\%) were in the 11-20 years age group. There were a total of 3 pediatric patients $(5.88 \%)$ and $7(13.72 \%)$ patients were above 60years. Fever with chills, rigors and headache were the commonest presenting complaints. These findings are also comparable to study conducted by Sethi Bhavna et al, which showed $16 \%$ patient were in the 11-20 years age group and $26(13 \%)$ of pediatric patient. ${ }^{13}$

As the malarial parasites mainly infect erythrocytes, anemia is frequently observed in malaria. It arises from the combination of hemolysis of the infected red blood cells (RBCs) by the parasite and suppression of erythropoiesis. The mechanisms of red cell destruction include reticuloendothelial hyperplasia, reduced deformability of infected red cells, membrane changes and immune mechanisms. ${ }^{14}$ From the 51 cases of malaria, anemia was observed in 25 patients $(49.01 \%)$. This study showed statistically significant species variation in causing anemia $(\mathrm{P}$ value $<0.05$ ). In this study $80 \%$ of patients with P.falciparum and $41.45 \%$ of patients with P.vivax infection had anemia. Majority 59.02\% and $42.43 \%$ of the patients had mild and moderate anemia respectively, while only $20 \%$ had severe anemia. Incidence of anemia in P.vivax infection in this study is $41.45 \%$ which is comparable with the study carried out by Rajini CY et al, which showed $40.74 \%$ in P.vivax infection. ${ }^{12}$ Various studies, have reported on the occurrence of anemia in increased frequency in falciparum malaria, it is also observed in this study. This study showed incidence of anemia in $89.7 \%$ of P.falciparum infection which is comparable with study conducted by Agravat and Dhruva showed 93\% cases of anemia in P. falciparum infection. ${ }^{15}$

This could be attributed to the differential preference of the malarial parasites to erythrocytes of different ages. While P.vivax infects reticulocytes or young RBCs, P.falciparum can attack erythrocytes of all ages. Also, in falciparum malaria, destruction of both parasitized and no parasitized red blood cells occur. Lower peripheral parasitemia, increased activation of host inflammatory immune response and increased deformability of infected erythrocytes leading to reduced cytoadherence in microvasculature are the other factors cited for the benign pathology in P.vivax malarial infection. ${ }^{15}$ 
Thrombocytopenia is a frequent finding in acute falciparum malaria and is reported in various studies. The various mechanism postulated are splenic pooling, immune mediated lysis by generation of anti-platelet antibodies, oxidative stress, causing premature platelet death and bonemarrow dyspoeisis. In this study, out of 51 cases of malaria 48 cases had thrombocytopenia. This study showed statistically significant species variation in causing thrombocytopenia ( $\mathrm{P}$ value $>0.05$ ). In this study thrombocytopenia is observed in $90 \%$ of P.falciparum and $95.11 \%$ of P.vivax infections. Majority of patients have mild to moderate thrombocytopenia, which is $33.33 \%$ and $35.23 \%$ respectively. Severe thrombocytopenia is observed in $25.49 \%$ of cases. Incidence of thrombocytopenia in P.vivax infection is $95.11 \%$ which is comparable with study carried out by S. Apte et al, which showed $85 \%$ of cases with P.vivax infection. Incidence of thrombocytopenia in P.falciparum infection is $90 \%$ in this study, which is comparable with study carried out by Horstmann et al. ${ }^{17}$ which showed $85 \%$ of cases with P.falciparum infection. But few studies reported slight lower incidence of thrombocytopenia like $40 \%$ and 59\%. There was no significant difference in severity between species. The vivax malaria is commonly associated with mild hematological abnormalities. Although sever thrombocytopenia is commonly reported to be associated with Plasmodium falciparum infection and has been reported to occur in patients co infected with both Plasmodium falciparum and Plasmodium Vivax, its occurrence has been rarely reported in cases of Plasmodium vivax malaria. ${ }^{16}$ The precise mechanism behind thrombocytopenia, however, remains unclear. Both the immunological as well as the nonimmunological destruction of platelets have been implicated. Decreased thrombopoiesis has been ruled out, because platelet forming megakaryocytes in the marrow are usually normal or increased. Other postulates include peripheral destruction and the consumption of platelets. ${ }^{16}$ Immune complexes which are generated by the malarial antigen lead to the sequestration of the injured platelets by the macrophages in the spleen. This is supported by the finding that malaria patients have elevated levels of platelet-bound $\mathrm{IgG}^{17,18}$ Some investigators have suggested disseminated intravascular coagulation as a major mechanism in malaria but others have found no evidence of disseminated intravascular coagulation in any of their patients, including those with severe thrombocytopenia. Thrombocytopenia is reported to be present in both P. falciparum and P.vivax infections. Many of the cases in our study are vivax malaria infection. A good tolerance of low platelet counts is well known in malaria. This could be explained by platelet activation and an enhanced agreeability. In most of the studies, thrombocytopenia was neither associated with the severity of the disease or death in malaria. It usually disappears with the treatment of the disease and requires no treatment for itself. ${ }^{19}$

In this study leucopenia is observed in 9 patients out of 51. This study showed no statistically significant species variation in causing leucopenia ( $\mathrm{P}$ value $>0.05)$. Leucopenia is observed in $10 \%$ cases of P.falciparum and $19.51 \%$ of P.vivax infection, which is comparable with study of F.Ellis McKenzie et al ,which showed $10.7 \%$ cases of $P$. falciparum and $15.2 \%$ cases of P.vivax infections ${ }^{20}$. In comparative study of Ranjini CY et al. ${ }^{12}$ Leukopenia and neutropenia primarily reflects the state of hypersplenism.

Laboratory diagnosis of malaria is based upon the demonstration of the malarial parasite on microscopy which requires technical expertise and repeated smear examinations. Hematological abnormalities are considered a hallmark of malaria and statistical analyses have shown that many of these hematological values may lead to an increased clinical suspicion for malaria, thus initiating a prompt institution of specific therapy even in the absence of a positive smear report for malaria. A variety of hematological alterations like progressively increasing anemia, thrombocytopenia, leukocytosis or leukopenia have been reported in cases of malaria. ${ }^{22}$

Our present results add to the strong but neglected case for improving the standard methods of 
malaria microscopy.

\section{Conclusion}

Although these hematological alterations in malaria are not new to the subject, this data adds more detailed information to limited knowledge, especially from these highly affected rural and suburban zones. Amongst haematological parameters studied, frequency of alteration in platelet count and hemoglobin are more pronounced compared to total WBC count in patients of malaria.

Species variation is seen in severity of thrombocytopenia. Majority patients have mild to moderate thrombocytopenia. Severity of thrombocytopenia is more in P.falciparum infection than P.vivax infection. Species variation is seen in severity of anemia. Anemia is more severe in patients P.falciparum infection compare to P.vivax infection .Species variation is also observed in severity of leucopenia. Mild to moderate leucopenia is more observed in P.vivax infection .

\section{References}

1. "Malaria Fact sheet N 94" WHO. March 2014. Retrived 28 Aug 2014.

2. Taylor-Robinson AW. Immunoregulation of malarial infection: balancing the vices and virtues. Int $\mathrm{J}$ Parasitol 1998 Jan;28(1):135-148.

3. Das LK, Pan SP. Clinical manifestation of severe form of P.falciparum malaria in Koraput district of Orissa state, India. J Vector Borne Dis 2006;43:104-143.

4. Kakar A, Bhoi S, Prakash V, Kakar S. Profound thrombocytopenia in plasmodium vivax malaria. Diagn Microbial Infect Dis. 1999; 35:243-44.

5. Niazi GA. Hematological aspect of malaria in a population based hospital Saudi Arabia. J Egypt Soc Parasite 1995; 25:787-93.

6. Sen R, Tewari AD, Sehgal PK, Singh U, Sikka R, Sen J. Clinico-haematological profile in acute and chronic P.falciparum malaria in children. J Com Dis. 1994;
26:31-38.

7. Rojanasthien S, Surakamolleart V, Boonpucknavig S, Isarangkura P. Hematological and coagulation studies in malaria. J Med Assoc Thai 1992; 75 Supl 1:190-194.

8. Facer CA. Hematological aspects of malaria, In: Infection and Hematology. Oxford: Butterworth Heinmann Ltd., 1994. P. 259-94

9. Perrin LH, Mackey LJ, Miescher PA. The hematology of malaria in man. Semin Hematol 1982 Apr;19(2):70-82.

10. Dacie, S.J and Lewis, S.M.: Reference ranges and normal values, Practical Hematology, $10^{\text {th }}$ edition, U.K., Churchill Livingstone Pub., 14-17 (2006).

11. Ranjini CY, Roopa Murgod et al. comparison of hematological parameters between Vivax and Falciparum malaria. Int J Pharm Bio Sci 2012 Oct; 3(4): (B) $1120-1128$.

12. Sethi Bhavna, Parasitemia and Hematological Alterations in Malaria: A Study from the Highly Affected Zones.

13. Hematological changes in malaria: A comparative study, Dr Shamim Akhtar; Agravat AH, Dhruva GA., Hematological changes in patients of malaria. J Cell Tissue Research, 10(3):2325-2329, (2010).

14. Saleem Ahmed Khan, Waqar Ali et al. Platelet count in malaria. Pak J Pathol 86 2008; 19(3): 86-88.

15. Horstmann R.D., M.Dietrich, U.Bienzle and H. Rasche,2005. Malaria induced thrombocytopenia. Annals of Hematol;42(3):157-64.

16. Makkar RPS, Mukhopadhyay S, Monga A, Gupta A. Plasmodium vivax malaria presenting with severe thrombocytopenia. Braz J Infect Dis.2002; 6:263-65.

17. Faseela T.S, Ronald A. Roche, Anita K.B., Chaitra S Malli, Yashwanth Rai.Diagnostic value of Platelet Count in Malaria. Journal of Clinical and Diagnostic Research. 2011 
June, Vol-5(3): 464-466.

18. White Blood Cell Counts and Malaria, F. Ellis McKenzie

19. Dr. Kinjal Mendapara, Journal of Hematological Profile in Malaria patients coming to a Tertiary Care Center in Central Gujarat 2012 June, Page no. 12-13.

20. Tangpukdee N, Duangdee C, Wilairatana P, Krudsood S. Malaria diagnosis: a brief review. Korean J Parasitol 2009 Jun;47 (2):93-102.

21. Koltas IS, Demirhindi H, Hazar S, Ozcan K. Supportive presumptive diagnosis of Plasmodium vivax malaria. Thrombocytopenia and red cell distribution width. Saudi Med J 2007 Apr; 28(4):535-539.

22. Dr. Kinjal Mendapara, Journal of Hematological Profile in Malaria patients coming to a Tertiary Care Center in Central Gujarat 2012 June, Page no. 12-13. 